\title{
Sociología: Lugar de experiencia e intervención profesional
}

\author{
Entrevista a Justino Gómez de Benito* por \\ Mariela Delgado**
}

Viernes 6 de noviembre del $2020^{1}$.

Mariela Delgado: Buenas tardes profesor. En primer lugar, le quiero agradecer por ser parte de un nuevo número de la Revista Temas Sociológicos. Como equipo estamos muy contentos por su participación y hace mucho tiempo que estábamos organizando esta entrevista. Estamos felices de poder contar con su experiencia y testimonio.

Justino Gómez de Benito: Está bien. Yo en alguna medida también lo esperaba. Aún más considerando los 25 años de la Revista Temas Sociológicos.

Mariela Delgado: ¿Cuál fue el primer acercamiento que tuvo usted con la sociología?

Justino Gómez de Benito: A ver. Hay que situarnos en España, finales de los años 60, donde no existía la carrera de Sociología; pero donde empezaron a llegar sociólogos a España, gente que estudió Sociología en Estados Unidos. Yo me encontré con tres amigos curas que habían hecho sus posgrados en Sociología y que después han sido personajes y profesores en Madrid. Entonces, yo me los encontré allí y eso me motivó porque, además, el contexto de España en ese momento era de

\footnotetext{
* Español. Doctor en Sociología por la Universidad Católica de Lovaina, Bélgica. Académico de la Escuela de Sociología, Universidad Católica Silva Henríquez, Santiago de Chile, Chile.E-mail: jgomez@ucsh.cl

** Chilena. Licenciada en Sociología, Universidad Católica Silva Henríquez, Diplomada en Participación Política de la Mujer, Universidad Alberto Hurtado. Asistente editorial de la Revista Temas Sociológicos, Escuela de Sociología, Universidad Católica Silva Henríquez, Santiago de Chile, Chile. E-mail: marielandelgadosilva@gmail.com

1 Entrevista realizada en el marco del aniversario №25 de la Revista Temas Sociológicos.
} 
mucho trabajo; la consideración del cambio social y político se estaba tratando de implementar o que se estaba viviendo en España. El tema de lo social era muy fuerte, también en el campo socio-religioso en el que yo me movía en España.

Mariela Delgado: Usted no estudió Sociología en España.

Justino Gómez de Benito: No, estudié Historia, porque en ese momento mi idea era orientarme hacia la Pedagogía en Historia. Bueno, en España no había Pedagogía, había que ser licenciado en Historia, así empecé en la universidad, pero fue en Chile donde empecé a estudiar Sociología.

Mariela Delgado: ¿Se vino con esa motivación, de estudiar Sociología?

Justino Gómez de Benito: O sea, intentarlo, claro. Vinculado con el compromiso social. En ese momento yo estaba vinculado en temas de educación; tenía el compromiso de trabajar como profesor aquí en Chile, primero en Linares y después en Santiago.

Mariela Delgado: Qué interesante eso, porque tuvo el contraste de estar en Linares - que es pequeño-y estar luego en Santiago, la capital.

Justino Gómez de Benito: Claro, sobre todo en contextos de pobreza que era lo que a mí me interesaba, a eso vine, a trabajar en vinculación y superación de la pobreza. Ese fue mi primer trabajo en la educación, en Linares. Ahí me hice cargo de ver toda la problemática del país: eran los años 70, las elecciones, salió Allende y me vine a Santiago a estudiar Sociología en el año 71.

Mariela Delgado: ¿En qué contexto se desarrolló usted cómo sociólogo?

Justino Gómez de Benito: Está el proceso de cambio de toda la expectativa de la Unidad Popular, para mí, estudiar Sociología en ese momento significaba vincularme con todo el proceso y comprender y aportar al cambio profundo y radical que se estaban implementando en el país. Entonces, ese era el nexo: investigar, saber y trabajar en el cambio social, político y cultural que estábamos viviendo en ese momento.

Primero, estuve en la Universidad de Chile, dos años. Ahí el enfoque era fundamentalmente marxista, entonces, mucho trabajo sobre Marx 
y mucho trabajo metodológico y estadístico. Había una complementación muy fuerte de ambas cosas. Teníamos profesores famosos como Enzo Faletto, Marta Harnecker y otros personajes que pasaron por allá y, mucha, mucha estadística. Julián Rodríguez también fue un profesor que después tuve en la Pontificia Universidad Católica de Chile, PUC.

En el año 1973 empecé en la PUC. Ahí, la verdad, no me atrevo a decir muchos nombres, porque todos eran muy valiosos y significativos. Tengo muchos recuerdos de Manuel Antonio Garretón, joven profesor en aquella época y también de Carmen Castillo, que nos hizo Historia hasta el día 11 de septiembre. Después fui orientándome hacia la sociología de las organizaciones y los aportes de algunos profesores como: José Álvarez, Álvaro González, Pelagia Ortúzar, Darío Rodríguez. Fui orientándome en esa línea para darle un aterrizaje, porque una preocupación fundamental en ese momento era el campo profesional, es decir, hacia dónde orientar la sociología en el contexto que estábamos viviendo. Tenía intereses en esa línea y en sociología de la educación también había profesores interesantes y en metodología Muchinsky, que nos aportó mucho en metodología y políticas públicas.

Mariela Delgado: Profesor, cuando usted dice que en la Universidad de Chile había una orientación hacia la estadística y harto componente marxista. ¿Qué la diferenciaba de la PUC en cuanto a contenido?

Justino Gómez de Benito: En la Universidad de Chile el contenido era muy ideológico y con una pedagogía muy dogmática de transmitir conocimientos; era un marxismo poco aplicado. En cambio, en la PUC los profesores que tenían esos planteamientos usaban herramientas del materialismo histórico para llevarlo a procesos concretos de desarrollo, de cambio y políticos, es decir, había una proyección mucho más aplicada y a mí eso siempre me llamó la atención, la sociología más aplicada, no únicamente de los procesos macro y muy poco orientados a niveles medios y microsociales.

Mariela Delgado: ¿Hasta ahí eran la PUC y la Universidad de Chile las instituciones que impartían la carrera de Sociología?

Puede ser, no lo tengo muy claro. Parece que había Sociología en Concepción y, también empezó Sociología en la Universidad del Norte. Justamente tuve ahí un contacto con los estudiantes de esta últi- 
ma institución, donde Alejandro Guillier era presidente del centro de alumnos. Fuimos con un amigo para allá a hacer un trabajo y estuvimos trabajando con Bernardo Guerrero, con Alejandro Guillier y con otros personajes que hoy día están en la pantalla de la sociología y del campo público.

Mariela Delgado: Con harto trabajo de campo, porque salió a hacer sociología a otras regiones.

Justino Gómez de Benito: Sí, eso fue muy importante. Imagínate que en ese momento era como un desierto. En el año 77, en plena dictadura, estudiar Sociología en Santiago y compartirla con estudiantes de Antofagasta era muy relevante, porque si nosotros no sabíamos mucho para qué estudiábamos sociología, ellos tenían una expectativa mucho más negativa al respecto. Sin embargo, fueron capaces de darle una proyección regional y, en ese sentido, fue muy entretenida esa dinámica de encuentro y de diálogo de la experiencia de Santiago y de allá del norte. Salimos en el diario y todo fue interesante.

Mariela Delgado: ¿Cómo fue el contexto de la sociología de los años 80 ? ¿Qué valoración tiene usted de la sociología de esa época?

Justino Gómez de Benito: En los 80, dramático. Terminar de estudiar Sociología y no saber qué hacer. Ahora, ¿dónde está el problema mayor? En los que no teníamos una familia en el barrio alto. Aquí hay un tema muy importante: hoy día hay una democratización de la universidad, pero en ese momento ni en la Universidad de Chile, ni en la PUC yo tenía compañeros de la Plaza Italia para abajo². Entonces, para mí el desafío era vivir de la sociología, mientras que para gran parte de los egresados había una familia detrás que los respaldaba, que no era mi caso ni el caso de otros. Era dramático, para algunos más que para otros. En ese sentido, trabajar desde lo aprendido en sociología era un desafío muy importante. Ocurrió con varios compañeros que pudieron incorporarse a trabajos que eran poco menos que trabajos voluntarios, eso era, ser ayudante de muchos profesores, pero por bolitas de dulce; pero en contextos como FLACSO y la mis-

2 Se trata de una separación imaginaria que divide la capital entre la población con mayores recursos de los sectores de nivel socioeconómico medios o bajos. Así, el barrio alto (desde la Plaza Italia hacia el oriente) está separado del resto de las comunas (hacia el poniente) por este hito urbano. 
ma Universidad de Chile se podían permitir otros lujos que algunos no nos podíamos permitir y eso marca. Siempre está la idea de vivir de la sociología o de una sociología más profesional que permita sobrevivir; eso ha marcado mucho a las distintas generaciones. En la medida en que se ha ido democratizando el acceso a la universidad los estudiantes de Sociología están necesitando vivir de ella en la actualidad. Entonces, eso tensionó y sigue tensionando hoy día cada vez más. En ese momento había una sociología muy académica, pero de la cual podían vivir algunos nombres o personajes de FLACSO y otras instituciones, pero los demás, los nuevos sociólogos éramos ayudantes no más. Yo también fui ayudante en la PUC hasta muy viejito, éramos ayudantes veteranos; pero era algo imprescindible para poder mantenernos como pudiéramos.

Mariela Delgado: Pero siempre mantuvo su vínculo con la educación, porque el hecho de ser ayudante lo mantenía ligado siempre.

Justino Gómez de Benito: Claro, sí, siempre hasta bien entrado a los 80, hasta el año 86 fui ayudante de cursos en la PUC.

Mariela Delgado: Profesor y ahí cuando dice hasta el año 86. ¿Después de eso a dónde se fue?

Justino Gómez de Benito: A Bélgica, a hacer un doctorado. El hecho de estar trabajando vinculado con instituciones de iglesia también era un recurso que me permitió tener una beca de la Universidad Católica de Lovaina. Para otorgarla se consideraban no solamente variables académicas, sino también el compromiso de trabajo que yo venía realizando en Santiago en sectores populares; eso también era importante para adjudicar la beca.

Mariela Delgado: Profesor y usted ¿Qué recuerdos tienen de la formación de la Revista Temas Sociológicos?

Justino Gómez de Benito: Yo estaba en ILADES a la vuelta de Bélgica. Trabajé con un equipo de profesionales en asesoría, también en la Academia de Humanismo Cristiano y finalmente se abrió esta posibilidad de la Universidad Católica Silva Henríquez, UCSH —en ese momento Universidad Católica Blas Cañas-. Llegué y estuve a cargo de la Dirección de Investigación y después me pidieron que me hiciera cargo de la Escuela de Sociología y ahí me encontré con la revis- 
ta. Cuando llegué a la UCSH me presentaron lo que estaba haciendo Jorge Gibert, que me parecía que era una heroicidad - siempre me pareció-, que el tema de la revista era una heroicidad, por las condiciones con las que trabajaban. Cuando me hice cargo del Departamento era un cacho, pero gracias al trabajo de Miguel Urrutia y con su ayuda - porque la universidad institucionalmente ayudaba poco-, entre nosotros quisimos abrirle campo a los colegas, aunque era muy sacrificado y con muy poca posibilidad de cumplir con plazos, esa es la verdad. La labor ha sido muy incomprendida porque claro, hoy día las reglas del juego son otras, entonces haber trabajado de esa manera fue muy difícil, complejo y yo creo que incomprendido; sacar arriba la revista de esa manera fue muy injusto, porque nos sacamos la mugre todos y, como no cumplíamos los plazos, era mucha la carga negativa que llegaba.

Mariela Delgado: ¿Se refiere a la competencia con otras revistas de la disciplina? ¿la competencia entre colegas?

Justino Gómez de Benito: La competencia será ahora, pero en ese momento había muy poca. Había muy pocos espacios donde publicar en los años 90 e inicio del 2000. Éramos una buena alternativa, un buen servicio que le hacíamos a nuestro campo, a los colegas y por eso hasta sobraban artículos, porque de verdad que había mucho ánimo, mucha voluntad de cooperar -así lo entendíamos-; no había un espíritu de competencia, sino uno de colaboración.

Una cosa importante también es que nosotros entendíamos que, primero, la revista era un espacio para otros, pero después dijimos: "aprovechemos de publicar nosotros". Después hubo un cambio de criterio, entonces definimos que no correspondía que publicáramos en nuestra propia revista.

Mariela Delgado: En la misma línea, ¿cómo surge la Escuela de Sociología de la UCSH? ¿Qué motivación hubo de crear la carrera de Sociología en el 2000?

Justino Gómez de Benito: Bueno, coincidieron varias cosas. En la Universidad Católica Blas Cañas, antes de que yo llegara, ya se había hablado de la posibilidad de abrir una carrera de Sociología. Estaba Jorge Baeza y con el mismo Jorge Gibert ya habían hablado de proceso. Entonces, cuando yo llegué me sumé a algo que ya se había iniciado. A 
mí me correspondió después armarlo y darle forma, obviamente marcar un itinerario de elaboración, pensar la carrera ya en términos de proyecto concreto. Ahí fue cuando con Mario Sandoval nos vinculamos con nuestros viejos profesores de Lovaina, con el apoyo de la rectora de esa época, Hilda Chiang. Entonces, nos fuimos a Lovaina y estuvimos trabajando con el profesor Guy Bajoit y otros docentes que nos dieron orientaciones; vimos modelos de otras escuelas de Sociología allá en Lovaina y luego en Bruselas. Después, gracias a esa experiencia y a algunos contactos que teníamos con Franck Poupeau, discípulo de Pierre Bourdieu, nos pusimos en contacto con él. Entonces, le compartimos nuestro primer proyecto de carrera y él fue quien de alguna manera nos compartió, a propósito del tema del oficio del sociólogo, un texto que a juicio nuestro aquí en Santiago era muy rígido, como una única manera de hacer sociología. Entonces, conversando nos dijo: "ustedes pueden ir más lejos", eso significaba que nos daba cancha para abrir nuevos horizontes, nuevas maneras de hacer sociología. Ese ha sido para mí un panorama muy importante, una sociología en plural, no una única manera de hacer sociología.

Yo creo que durante un tiempo se entendió la sociología como una sociología académica, de escritorio, de intelectuales en la universidad, dejando el trabajo en terreno para los trabajadores sociales. Esa dicotomía yo no la he entendido nunca, eso lo vivimos en la PUC durante el primer año, esa experiencia de estudiar sociología desde la práctica, estudiando y trabajando, vinculados con espacios laborales, con experiencias de la Reforma agraria y otras, entonces, esa relación de cátedra y terreno a mí me pareció muy importante. Esa fue una de las motivaciones y planteamientos que conversamos con Bourdieu.

Mariela Delgado: ¿Usted considera que hubo una influencia de Pierre Bourdieu en el sello y el perfil de la Escuela de Sociología de la $\mathrm{UCSH}$ ?

Justino Gómez de Benito: Yo creo que sí, porque ahora mismo este último rediseño tiene un compromiso con la intervención social. Efectivamente va en la misma línea, es decir, recoge parte de esa intuición, aunque esté expresada de otra manera, pero ciertamente hay una coherencia: la formación de nuestros estudiantes no va orientada a ser investigadores de cátedra, sino a enfrentar problemáticas reales para la transformación social. 
Mariela Delgado: ¿Qué debates y temas estaban en el escenario general de la sociología en los años 2000?

Justino Gómez de Benito: En ese momento se cumplieron los 40 o 50 años de la FLACSO y hubo un debate muy enérgico debido a que Brunner planteó en su discurso el fin de la sociología. Justo estábamos nosotros pensando el proyecto de carrera cuando aparece esta bomba del fin de la sociología y de la polémica que se creó. Ahí es donde con más fuerza buscamos recursos, aprovechamos de invitar a Gabriel Salazar, incluso con el mismo Brunner luchamos contra este planteamiento, justo en la línea de buscar nuevos horizontes, nuevas identidades, nuevas maneras de ser sociólogo y eso se transformó, en el caso de nuestra Facultad de Ciencias Sociales, en un proyecto que incluyó las carreras de Trabajo Social, Psicología y Sociología. Es decir, las tres áreas nos involucramos en un proyecto de investigación acerca de la transformación del campo profesional, de las identidades profesionales y de la pedagogía para formar psicólogos, sociólogos y trabajadores sociales y nos enfocamos en un trabajo interdisciplinar. En ese momento yo fui decano y eso lo lideramos desde la decanatura.

Mariela Delgado: ¿Cuál es su percepción acerca del desarrollo de la sociología en Chile en los últimos 20 años?

Justino Gómez de Benito: Yo encuentro de verdad que ha habido una explosión que yo le llamo maravillosa y fructífera. Es tremendamente ilusionante ver la cantidad y la pluralidad de grupos que participan en los congresos de sociología - no solamente en ALAS, sino que en los nuestros, en los de Chile-, cómo han proliferado los temas y cómo conviven. Vuelvo a las relaciones de dominación, porque durante una época hubo mucha discriminación y por eso también se cortaron los congresos de sociología, porque no hubo capacidad de diálogo; durante los 90 hubo muy poca capacidad de diálogo entre sociólogos.

Mariela Delgado: ¿Pero eso estaba también relacionado con el clivaje político del momento?

Justino Gómez de Benito: También, claro, y con el posicionamiento en espacios de gobierno. Entonces, creo que la proliferación temática y la diversidad de hacer sociología ha sido tremendamente positivo. Por otro lado, la valoración de la dimensión de formación, es decir, cuando el rol de formadores recuperó su valor, recuperó su mérito y nos encon- 
tramos diciéndonos: "es muy importante formar sociólogos, juntémonos como escuelas de sociología, no como investigadores, sino como formadores y hagámonos cargo y responsabilicemos de formar buenos sociólogos para el futuro, para la sociedad". Creo que ahí hay un mérito muy importante en la capacidad de todas las escuelas, de otras generaciones; los que andaban peleados eran las viejas guardias, viejos sociólogos. En ese momento los viejos eran otros, ahora lo somos nosotros.

La proliferación de temáticas, su complementación, la diversificación de las sociologías, sociologías de todo tipo, la capacidad de conexión metodológica, tópicos macro y micro, sociologías cuantitativas y cualitativas. Es decir, la complementación y el reencuentro de muchos temas y metodologías que aparecieron en una época contradictoria y opuesta. Yo eso lo valoro mucho como parte de esa historia de la última década.

Mariela Delgado: ¿Qué han integrado las nuevas generaciones de sociólogos al desarrollo de nuestra disciplina?

Justino Gómez de Benito: Las nuevas generaciones han colocado nuevos temas y eso es muy interesante, porque quizá nosotros estábamos acostumbrados a que fueran otros, eran los profesores los que ponían los temas. Hoy día yo me doy cuenta que son ustedes los que ponen los temas, los que dicen lo que quieren investigar: yo quiero hacer mi tesis en esto. Entonces antes uno iba y decía: "Oiga profe, ¿puedo hacer la tesis con usted?", o sea, a la sombra de los profesores. Hoy día no, ustedes saben, están interesados e interesadas en tales temas y ustedes salen adelante, con apoyo metodológico, orientaciones, pero creo que eso es muy importante, que hay problemáticas más coyunturales, más contextuales, de nuevos movimientos, de la diversidad, mucho sobre las problemáticas que tienen que ver con las nuevas representaciones de las realidades y las identidades. Creo que eso le está dando mucha juventud a la sociología, mucho protagonismo a ustedes, porque fueron ustedes los que salieron a la calle, se la jugaron por los movimientos sociales, por las tribus urbanas, es decir, los jóvenes son los que han puesto los temas en la última década y nosotros estamos en la competencia por los recursos, buscando dónde publicar nuestros artículos; en eso andan los sociólogos en edad adulta. 
Mariela Delgado: A propósito de todos los movimientos sociales que han surgido, del estallido social de octubre del año pasado y el nuevo proceso constituyente que abre paso a nuestras transformaciones de la sociedad, ¿cuáles cree usted que son los principales desafíos de nuestra disciplina?

Justino Gómez de Benito: Uno de los desafíos es dar trabajo a los sociólogos. Ese es un desafío muy grande, lograr que la sociología sea un espacio profesional del cual podamos vivir, entonces hay una responsabilidad muy grande, de toda la sociedad y nuestra, de legitimarse. Para mí, ese es un compromiso de responsabilidad muy grande. Lo otro es hacernos cargo de los nuevos temas, saber interpretar lo que está pasando hoy, los nuevos acontecimientos. Siento que no hemos estado al nivel para comprender el estallido social, para comprender y decir palabras en este contexto de pandemia y sus desafíos, creo también que la convivencia de la cotidianidad, todo este ambiente tan crispado, tan incapaz de tolerarnos, creo que la sociología tiene que saber manejar contenidos, teorías que nos ayuden a vivir mejor. Construir ciudades más amables, más cercanas, hay muchos desafíos en ese plano.

Mariela Delgado: $i$ Cree usted que estas nuevas demandas y transformaciones sociales se verán reflejadas próximamente en las distintas escuelas de sociología, en el contenido y forma de difundir el conocimiento?

Justino Gómez de Benito: Yo creo que sí. Formalmente a los equipos que estamos en la formación se nos exige captar los cambios de la sociedad para transformar el perfil. No construimos el perfil de lo que se nos ocurre, sino que hay un sondeo para captar hacia dónde van las necesidades y las problemáticas para orientar la formación hacia un tipo de sociólogo con un perfil acorde con esas necesidades y que después se inserten. Entonces, en ese sentido desde el punto de vista formal, hay una exigencia de captar los nuevos temas.

Después, yo me doy cuenta a partir de las propuestas que llegan de los cursos optativos, de lo que ustedes como estudiantes piden, por ejemplo, optativos y posibilidades de cursos y, por otra parte, las ofertas de nuevos académicos que ofrecen temas muy interesantes y bien fundamentados. Hay una riqueza de alternativas y temáticas, de enfoques y complementación. 
Se me quedó algo en el tintero en relación con los desafíos. Hay un desafío muy importante que es la interdisciplinariedad. Yo contaba antes la experiencia positiva que teníamos en la Facultad de Ciencias Sociales, cuando trabajábamos en equipo con la carrera de Sociología y Trabajo Social y creo que después no ha sido posible ni en nuestra universidad. Se dan pocas experiencias de trabajo interdisciplinario, pero les hace bien a los estudiantes compartir con compañeros de otras disciplinas, eso le hace bien a la formación. Los problemas son muy complejos hoy día, entonces todo debe abordarse desde lo interdisciplinar, ya que así es también el campo laboral. 